\title{
Hypoxia and TP53 deficiency for induced pluripotent stem cell-like properties in gastrointestinal cancer
}

\author{
HIROMITSU HOSHINO $^{1}$, HIROAKI NAGANO ${ }^{1}$, NAOTSUGU HARAGUCHI ${ }^{2}$, \\ SHIMPEI NISHIKAWA ${ }^{1}$, AKIRA TOMOKUNI ${ }^{1}$, YOSHIHIRO KANO ${ }^{1}$, TAKAHITO FUKUSUMI $^{1}$, \\ TOSHIYUKI SAITO ${ }^{3}$, MIYUKI OZAKI $^{2}$, DAISUKE SAKAI ${ }^{2}$, TAROH SATOH ${ }^{2}$, HIDETOSHI EGUCHI $^{1}$, \\ MITSUGU SEKIMOTO $^{1}$, YUICHIRO DOKI ${ }^{1,2}$, MASAKI MORI $^{1,2^{*}}$ and HIDESHI ISHII ${ }^{1-3^{*}}$ \\ Departments of ${ }^{1}$ Gastroenterological Surgery, ${ }^{2}$ Frontier Science for Cancer and Chemotherapy, Osaka University \\ Graduate School of Medicine, Suita, Osaka 565-0871; ${ }^{3}$ Transcriptome Profiling Group, National Institute \\ of Radiological Sciences, Research Center for Charged Particle Therapy, Chiba 263-8555, Japan
}

Received October 14, 2011; Accepted December 1, 2011

DOI: 10.3892/ijo.2012.1346

\begin{abstract}
Induced pluripotent stem (iPS)-like cancer cells (iPC) by the introduction of defined transcription factors reduce the prevalence of the malignant phenotype of digestive system cancer cells, but the induction efficiency is low. The role of hypoxia and TP53 deficiency in iPC cell generation remain unclear. Cellular reprogramming was performed by retroviral infection with $O C T 3 / 4, S O X 2, K L F 4$ and $c-M Y C$ of wild-type HCT116 colorectal cancer cells and mutant TP53-deficient HCT116 cells. Cells were cultured in normoxia $\left(21 \% \mathrm{O}_{2}\right)$ or hypoxia $\left(5 \% \mathrm{O}_{2}\right)$ for 30 days after transduction, and the response to hypoxia and comparison of cellular proliferation, invasion and tumourigenesis before and after iPC cell generation were studied. iPC cell generation from wild-type HCT116 cells in hypoxia was approximately 4-times greater than in normoxia ( $\mathrm{p}<0.05)$, and TP53 deficiency increased conversion efficiency significantly in normoxia $(\mathrm{p}<0.05)$. Significant involvement of hypoxia-inducible factors was observed in an immature carbohydrate epitope, Tra-1- $60^{+}$, colony formation. Generated iPC cells exhibited multi-differentiation potential. Although the iPC cells in hypoxia exhibited reduced proliferation, invasiveness and tumourigenicity, TP53 deficiency in iPC cells resulted in
\end{abstract}

Correspondence to: Professor Masaki Mori, Department of Gastroenterological Surgery, Osaka University Graduate School of Medicine, Yamadaoka 2-2, Suita, Osaka 565-0871, Japan

E-mail: mmori@gesurg.med.osaka-u.ac.jp

Professor Hideshi Ishii, Department of Frontier Science for Cancer and Chemotherapy, Osaka University Graduate School of Medicine, Yamadaoka 2-2 (E21-19), Suita, Osaka 565-0871, Japan

E-mail: hishii@gesurg.med.osaka-u.ac.jp

*Contributed equally

Key words: cancer reprogramming, epigenome normalisation, hypoxia, tumour suppressor genes higher tumourigenicity than in wild-type cells. Both hypoxia and TP53 deficiency increase iPC cell generation. TP53 deficiency can also result in deleterious mutations, whereas hypoxia may impact molecular targets of epigenome normalisation.

\section{Introduction}

Although disruption of the normal differentiation process is an important component of tumourigenesis (1) and is involved in leukaemogenesis (2) and the formation of other malignancies (3), not much is understood about the reversibility of this process. In general, genetic alterations such as mutations (substitutions of nucleotide sequences), amplifications and deletions, as well as recurring chromosomal aberrations are irreversible, whereas epigenetic alterations can be modified by pharmacological agents that target components of the epigenetic machinery (4). Since epigenetic modifications, including DNA methylation and histone modifications, substantially contribute to the tumour cell phenotype, the number of potential therapeutic targets has increased (5). Epigenome normalisation is a potential therapeutic approach for cancer treatment in the clinical setting and in translational aspects of epigenetic research (5).

An important discovery that has been reported is that complete reprogramming can be achieved by the introduction of defined transcription factors, Oct4 (also known as Pou5f1), Sox 2, Klf4 and cMyc, from terminally differentiated somatic fibroblasts (6). Generation of induced pluripotent stem (iPS) cells is believed to require epigenetic modifications, but the precise mechanism is unknown (7). Recently, we showed that introducing defined factors in gastrointestinal cancer cells resulted in the acquisition of multi-differentiation potential, i.e. the gene expression profiles of mesoderm and ectoderm appeared in gastrointestinal cancer cells of endodermal origin [iPS-like cancer (iPC) cells] (8). Reprogramming of sensitised cancer cells in response to differentiation therapy suppressed tumourigenicity in vivo (8); this presumably involved reactivation of tumour suppressor genes at the CDKN2b-CDKN $2 a$ locus on chromosome 9p21 in humans (chromosome 4 in mice), a region that is frequently inactivated in cancer and 
is involved in the reduction of chemosensitivity (9). Together, these findings strongly suggest that the magnitude of epigenetic modifications using iPS technology may be sufficient to reverse the differentiation programme and lead to a multipotent state as well as contribute to the suppression of biologically malignant phenotypes in cancer cells.

Recent studies have shown that silencing or absence of p53 significantly increases iPS cell generation (10-12). Another study demonstrated that hypoxia enhance iPS cell generation in humans and mice (13). Since p53 activation characterises cancer cells that survive in hypoxia $(14,15)$, it is possible that reprogramming of cancer cells may be closely associated with the p53 and hypoxic pathways. Herein, we studied these pathways and demonstrated that although prolonged in vitro culture increased tumourigenic potential in TP53-deficient iPC cells, hypoxia and TP53 deficiency enhances iPC cell generation. This suggests that TP53 deficiency functions as a two-edged sword and that TP53 is a candidate molecular marker for predicting the biological behaviour of reprogrammed cancer cells.

\section{Materials and methods}

Cell lines and culture. Wild-type (wt) and TP53-deficient colorectal cancer (CRC) cell lines, wt HCT116 cells and its homologous-recombination mutant, HCT116 p53-deficient (null) cells, were donated by Dr Bert Vogelstein (Johns Hopkins University, Baltimore, MD, USA), and other cancer cell lines were obtained from DS Pharma Biomedical Co., Ltd. (Osaka, Japan). Cell lines were maintained in Dulbecco's modified Eagle's medium (DMEM; Nacalai Tesque, Kyoto, Japan) supplemented with $10 \%$ foetal bovine serum (FBS) at $37^{\circ} \mathrm{C}$ in a 5\% humidified $\mathrm{CO}_{2}$ atmosphere. Plasmids were purchased from Addgene (Cambridge, MA). Transfectants were grown in DMEM supplemented with 10\% FBS and puromycin $(2 \mu \mathrm{g} /$ $\mathrm{ml})$ and subsequently incubated in specific culture conditions as described previously (8). All retroviral transfections were performed using ViraDuctin Transduction kit (Cell Biolabs, San Diego, CA). Transfections with lentivirus were performed using the Virapower Lentiviral Packaging mix (Invitrogen, Carlsbad, CA). In brief, cancer cell lines were transfected with plasmids at a concentration of $4 \mu \mathrm{g} / \mu \mathrm{l}$ using Lipofectamine 2000 (Invitrogen) and incubated in glucose-free Opti-MEM (Invitrogen). These transfected cell lines were cultured in $21 \%$ or $5 \% \mathrm{CO}_{2}$. All experiments were performed at $50-70 \%$ cell confluence and results were confirmed in at least three independent experiments. All-in-one type fluorescence microscope (BZ-8000; Keyence, Osaka, Japan) with digital photographic capability was used to visualise cells at several magnifications. In the proliferation assay, growth rates of the cultured gastrointestinal cancer cell lines were measured by counting cells using Celltac (Nihon Kohden Co., Tokyo, Japan).

$R N A$ preparation and real-time reverse transcriptionpolymerase chain reaction ( $q R T-P C R)$. Total RNA was prepared using TRIzol reagent (Invitrogen). RT was performed using SuperScript III RT kits (Invitrogen). To confirm PCR amplification, 25-35 cycles of PCR were performed using a PCR kit (Takara, Kyoto, Japan) on the GeneAmp PCR System 9600 (PE Applied Biosystems, Foster City, CA) in the following conditions: $95^{\circ} \mathrm{C}$ for $10 \mathrm{sec}, 60^{\circ} \mathrm{C}$ for $10 \mathrm{sec}$ and $72^{\circ} \mathrm{C}$ for $60 \mathrm{sec}$. An 8- $\mu \mathrm{l}$ aliquot of each reaction mixture was size-fractionated in a $1.5 \%$ agarose gel and visualised by ethidium bromide staining. To confirm RNA quality, PCR amplification was performed for $G A P D H$ using specific primers. For quantitative assessment, the gene expression was evaluated by qRT-PCR using a LightCycler TaqMan Master kit (Roche Diagnostics, Tokyo, Japan) for cDNA amplification of specific target genes. The expression of mRNA copies was normalised against GAPDH mRNA expression.

Reagents and antibodies. Antibodies against Nanog, Ssea-4, Tra-1-60, Tra-1-81 and Tra-2-49 (Chemicon International Inc., Temecula, CA) were used for immunocytology.

Invasion assay. Cell invasion was analysed using the CytoSelect assay kit according to the manufacturer's protocol (Cell Biolabs). Cells $\left(1.0 \times 10^{5}\right)$ in DMEM were placed on $8.0-\mu \mathrm{m}$ pore-size membrane inserts in 96-well plates, and DMEM with $10 \%$ FBS was added to each well. After $24-\mathrm{h}$ incubation at $37^{\circ} \mathrm{C}$ in $5 \% \mathrm{CO}_{2}$, non-invading cells were removed from the top of the membrane chamber and cells on the underside of the membrane were completely dislodged by tilting the membrane chamber in cell detachment solution (Cell Biolabs). Lysis buffer/CyQuant GR dye solution (Cell Biolabs) was then added to each well, and fluorescence was determined at 480 or $520 \mathrm{~nm}$ using a plate reader to estimate the number of cells that had invaded the undersurface of the membrane. Each assay was performed in triplicate.

Tumourigenicity. Cells were subcutaneously inoculated into NOD/SCID mice. The tumour diameter and size were estimated using the following formula: size $=(\text { length })^{2} \mathrm{x}$ (width) $/ 2$

Statistical analysis. For continuous variables, results are expressed as means \pm standard errors. The relationship between the gene expression level and cell count was analysed by Chi-square and Wilcoxon rank tests. All data were analysed using JMP software (SAS Institute, Cary, NC). Differences with $\mathrm{p}<0.05$ were considered statistically significant.

\section{Results}

Lentiviral- and retroviral-mediated iPS factor gene transfer. The expression profile of ES-like genes is reportedly associated with aggressive phenotypes observed in solid tumours (16). Considering that reprogramming induces the endogenous expression of ES-like genes, it is assumed that the expression of endogenous genes may be involved in reprogramming and some cancer cells with a relatively higher expression of endogenous ES-like genes may be sensitive to reprogramming induction. We quantitatively studied the expression of endogenous immature NANOG by qRT-PCR (data not shown), because $N A N O G$ is not included in exogenously mediated reprogramming vectors in the present experiment but is relevant to the immature status of iPS cells $(6,17)$. The NANOG expression level in HCT116 p53 null cells was equal to that in PANC-1 cells, but compared with that in NTERA teratoma cells it was $\sim 20 \%$ greater, while the expression in wt HCT116 cancer cell lines was relatively low (data not shown). We thus selected two cells each from wt HCT116 cells, its derivative 
A
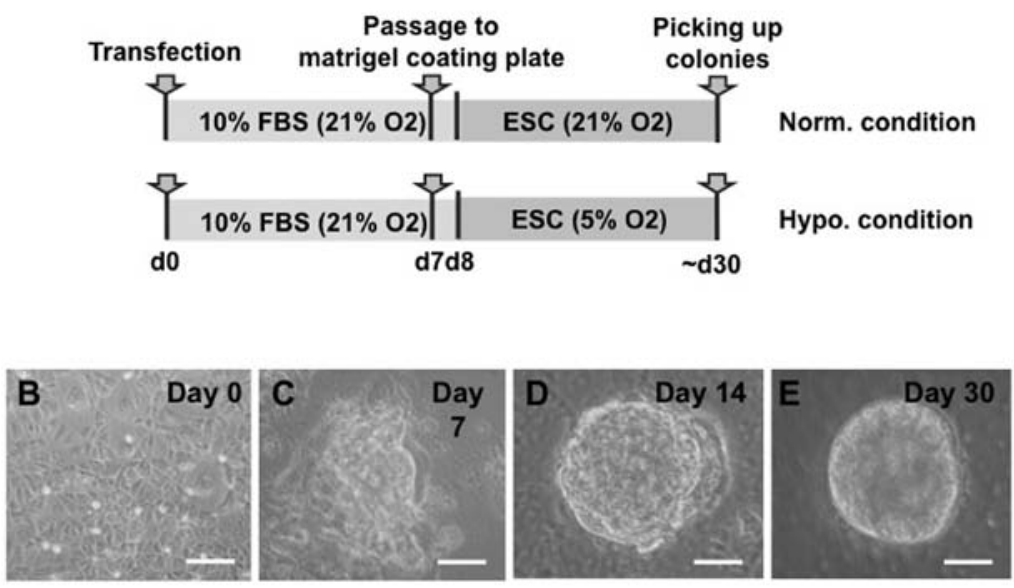

Figure 1. Retroviral- and lentiviral-mediated gene transfer of four iPS factor genes in gastrointestinal cancer cells. (A) Schematic representation of the experiment showing normoxia and hypoxia. (B-E) Morphological changes in iPC cells derived from pancreatic cancer wt HCT116 cells. (B) Day 0, (C) day 7, (D) day 14, (E) day 30 after transfection. Scale bar, $100 \mu \mathrm{m}$.

A

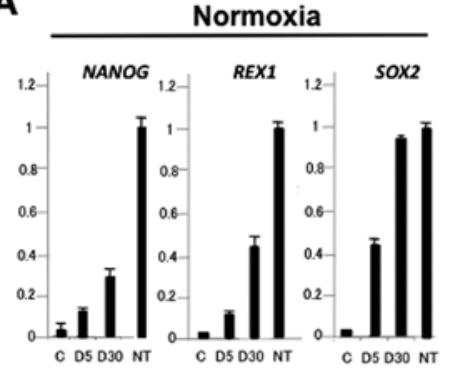

B
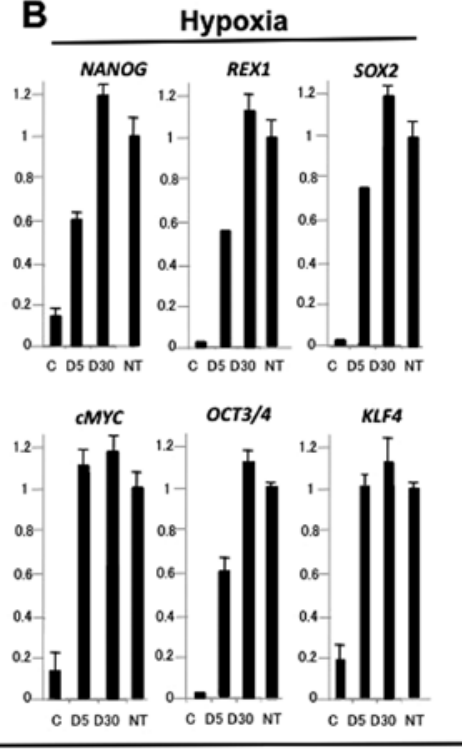

HCT-116-wt

Figure 2. Exogenous and endogenous gene expression by iPS cells after retroviral- and lentiviral-mediated gene transfer in wt HCT116 cells. After viral vector-mediated gene transfer of the four defined factor genes $c-M Y C, S O X 2, O C T 3 / 4$ and $K L F 4$, the expression of all exogenous and endogenous genes was assessed in normoxia (A) or hypoxia (B) on days 5 and 30 by qRT-PCR using specific primers.

line HCT116 p53 null cells and PANC-1 cells for comparison in subsequent experiments.

For the transfer of iPS factor genes, we infected cells on day 0 through lentiviral-mediated transfer of murine retroviral receptors followed by retroviral-mediated gene transfer of the four defined factor genes $c-M Y C, S O X 2, O C T 3 / 4$ and $K L F 4$. On day 7, cells were transferred to ES culture medium in either hypoxia $\left(5 \% \mathrm{O}_{2}\right)$ or continuous normoxia $\left(21 \% \mathrm{O}_{2}\right)$ (Fig. 1A). The number of cells increased on day 7 and clear round colonies were formed by day 30 (representative data are shown in Fig. 1B-E). These induced cells were morphologically similar or indistinguishable from iPC cells derived from other gastrointestinal cancer cells (8) and were similar to iPS cells derived from terminally differentiated normal cells (7).
The expression in wt HCT116 and HCT116 p53 null cells of four exogenously introduced transgenes and endogenous genes in normoxia and hypoxia were analysed by qRT-PCR (Fig. 2, and data not shown). On day 5, the expression levels of exogenously introduced c-MYC, SOX2, OCT3/4 and KLF4 in wt HCT116 cells were $40 \%-80 \%$ and $>60 \%$ and those of REX1 and NANOG, which were not introduced as transgenes, were $\sim 10$ and $60 \%$ of those in teratoma NTERA cells in normoxia and hypoxia, respectively (Fig. 2). These results suggested that compared with normoxia, hypoxia stimulates endogenous expression of ES-like genes, such as REXI and $N A N O G$, and the relevance of this result was appreciable in assessment of four exogenously introduced transgenes and endogenous genes. 
A

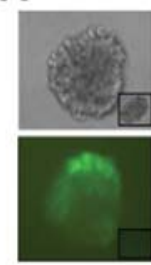

HCT-116-wt
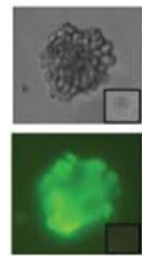

HCT-116-p53null
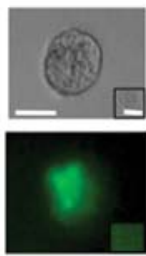

PANC-1
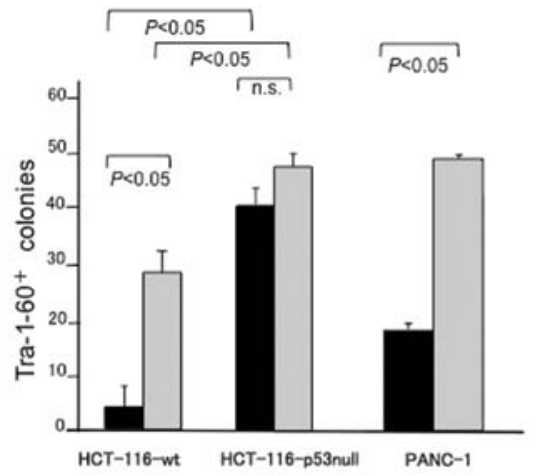

B

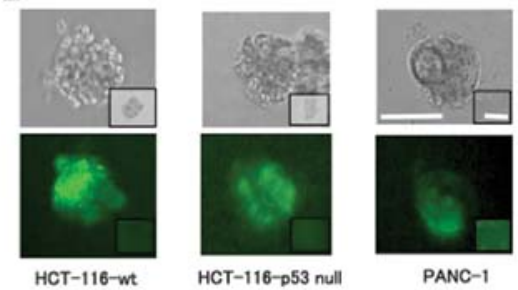

HCT-116-wt

PANC-1

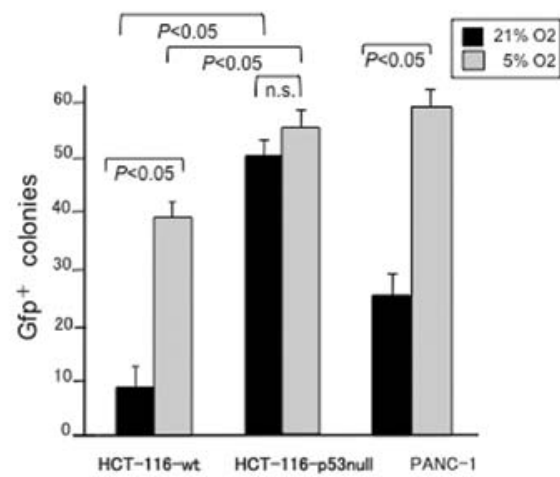

Figure 3. Effect of hypoxia on TP53-deficient cancer cells. The effects of reprogramming of wt HCT116, HCT116 p53 null and PANC-1 cells are shown by (A) immunostaining with anti-Tra-1-60 antibody or (B) observation of GFP-positive spheres under a fluorescence microscope on day 30. Reprogramming efficiency (percentage of Tra-1-60- or GFP-positive cells) in hypoxia $\left(5 \% \mathrm{O}_{2}\right)$ was higher than in normoxia $\left(21 \% \mathrm{O}_{2}\right)$. p-values are shown for each comparison. Scale bar, $50 \mu \mathrm{m}$.

On day 30 , the expression levels of exogenously introduced REXI and NANOG were $30-40 \%$ and $>100 \%$ and those of the endogenously introduced $c-M Y C$, SOX 2 and $O C T 3 / 4$ were $>80 \%$ of those in teratoma NTERA cells in normoxia and hypoxia, respectively (Fig. 2). By utilising specific primers, the assessment of exogenous expression levels on day 30 indicated that all transgenes in wt HCT116 cells had decreased to undetectable levels in normoxia and hypoxia, except for the expression levels of $c-M Y C$ in normoxia and hypoxia and KLF4 in hypoxia, all of which persisted (data not shown). By day 30, the expression levels of all transgenes in HCT116 p53 null cells had decreased to undetectable levels in normoxia and hypoxia, except for the expression levels of $c-M Y C$ and $K L F 4$ in normoxia and hypoxia that persisted, and the expression levels were higher in hypoxia (data not shown). The overall trend of decreased exogenous gene expression levels is probably due to silencing of the introduced genes (7). Our data indicate that reprogramming factors elicited the specific activation of immature endogenous ES-like genes.

High expression of ES-like genes in hypoxia and TP53deficient condition in iPC cells derived from gastrointestinal cancer. After the introduction of iPS factors, compared to the expression levels in wt HCT116 cells in normoxia and hypoxia, the expression levels of an immature carbohydrate epitope, Tra-1-60, in iPC cells derived from HCT116 p53 null cells was 8 -times and 1.6-times greater ( $\mathrm{p}<0.05$, Fig. $3 \mathrm{~A})$, while those in PANC-1 cells was 2.4-times greater in hypoxia $\left(5 \% \mathrm{O}_{2}\right)$ than in normoxia $\left(21 \% \mathrm{O}_{2}\right)(\mathrm{p}<0.05$, Fig. $3 \mathrm{~A})$. These data indicate that p53-defiency induces reprogramming in normoxia, and to a lesser extent, in hypoxia, suggesting that the factors downstream of p53 signalling may be involved in increased reprogramming efficiency in hypoxia. On day 30, the increased expression of other immature carbohydrate epitopes and proteins, namely Tra-2-49, Tra-1-60, Tra-1-81, Ssea4 and Nanog, was confirmed by immunocytochemistry after the introduction of defined factors (data not shown).

For semi-quantitative analyses of $N A N O G$ promoter activity, the $N A N O G$ promoter fusion green fluorescent protein vector was co-transfected in iPC cell generation. The tracing study of $N A N O G$ promoter activity in iPC cells derived from HCT116 p53 null cells indicated that, compared with wt HCT116 cells, the endogenous NANOG promoter was activated after reprogramming in normoxia (5-times, $\mathrm{p}<0.05$, Fig. $3 \mathrm{~B}$ ), and to a lesser extent in hypoxia (1.3-times, $\mathrm{p}<0.05$, Fig. 3B). Similarly, NANOG promoter was activated in hypoxia in PANC-1 iPC cells (2.2-times, p $<0.05$, Fig. 3B). The data are consistent with the notion that reprogramming efficiency is increased by $\mathrm{p} 53$ deficiency and hypoxia in a common pathway. Although the observed $N A N O G$ reporter activity may reflect endogenous activity at a basal level (data not shown), the expression of Tra-1-60 seems to be more specific to reprogramming induction $(6,17)$. We thus used Tra-1-60 to assess reprogramming in the following studies.

Previous studies indicated that the hypoxia-inducible factor (Hif) pathway plays a role in metabolic regulation of cancer, as in the p53 pathway (15). Thus, to determine whether Hif affects the downstream cellular response to reprogramming induction, we co-transfected cells with hydroxylation-defective active mutants HIF1A-P402A/P564A and HIF2A-P405A/P531A, which lack proline residues within the oxygen-dependent degradation domain. These residues are necessary for the interaction with von Hippel-Lindau tumour suppressor protein (pVHL) and their substitutions increase the stability of Hifs, because they resist proteasome-dependent degradation (15). qRT-PCR indicated that transgenes were detected for at least 5 days after transfection, which is the critical timeframe for reprogramming events involved in iPS generation $(6,17)$. The Tra-1-60 expression data indicated that the introduction of 


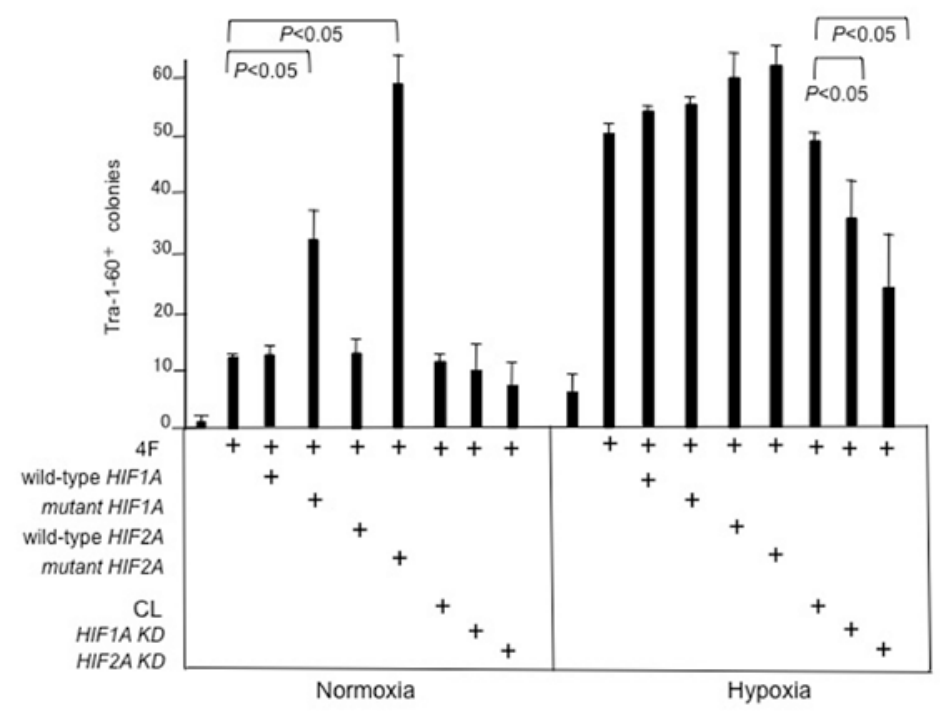

Figure 4. Efficiency of reprogramming induced by co-transfection with iPS factors and mutant $H I F$ in wt HCT116 cells. Number of Tra-1-60 ${ }^{+}$and GFP ${ }^{+}$clones as percentage of colonies on day 30 after gene transfer are shown. 4F, four factor genes (c-MYC, SOX2, OCT3/4 and KLF4); mutant HIF1A, the hydroxylationdefective mutant $H I F 1 A-P 402 A / P 564 A$ lacking proline residues that are necessary for the interaction with pVHL and are refractory to proteasome-dependent degradation; mutant HIF2A, the hydroxylation-defective mutant HIF2A-P405A/P531A; HIF1A KD, lentiviral shRNA knockdown of HIF1A; HIF2A KD, lentiviral shRNA knockdown of the HIF2A; CL, lentiviral shRNA against luciferase as a control. p-values are shown for each comparison.
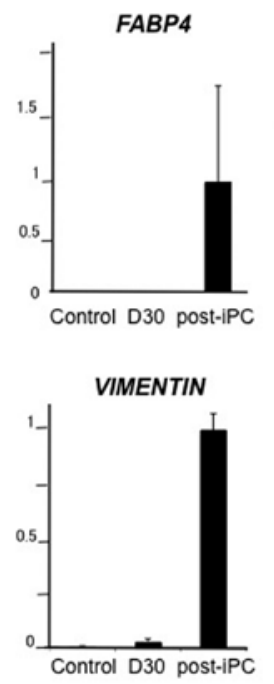

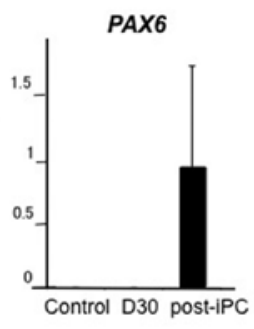

PDX1

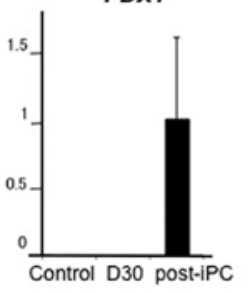

HCT-116-wt

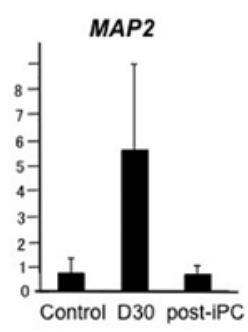

KRT19

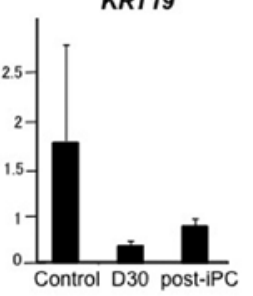

Control D30 post-iPC
Figure 5. Multi-differentiation potential of iPC cells after reprogramming After generating iPC cells from wt HCT116 cells in hypoxia $\left(5 \% \mathrm{O}_{2}\right)$, the expression levels of FABP4, PAX6, MAP2, VIMENTIN, PDX1 and KRT19 were analysed by qRT-PCR on day 30 (iPC; D30) or after subsequent differentiation-induction culture for 20 days (post-iPC).

HIF2A-P405A/P531A mutants, but not wild-type HIF2A, increased reprogramming efficiency in normoxia (4.8-times, $\mathrm{p}<0.05$, Fig. 4), whereas the introduction of HIFlA-P402A/ P564A mutants, but not wild-type HIF1A, increased reprogramming efficiency (2.7-times, $\mathrm{p}<0.05$, Fig. 4 ).

In hypoxia, the expression of Tra-1-60 was increased regardless of whether wild-type or mutant HIFIA and HIF2A were co-transfected, indicating that hypoxia attenuated the effect of mutations in Hif proteins and that the Hif pathway plays a role in the increased reprogramming efficiency of cancer cells. To confirm these indications, we performed a knockdown experiment. The lentiviral shRNA transduction system indicated that knockdown lasting for at least 5 days resulted in reduced generation of Tra-1- $60^{+}$cells in hypoxia. In other words, reprogramming efficiency was decreased after $H I F 2 A$ knockdown $(0.5$-times, $\mathrm{p}<0.05)$ and HIFlA knockdown $(0.75$ times, $\mathrm{p}<0.05$ ), but the knockdown effect was not detected in normoxia, supporting the notion that the Hif pathway plays a role in the efficiency of iPC cell generation in hypoxia. This suggests that the effect of hypoxia can be explained at least partially by the activation of the Hif2a pathway, and increased expressions in hypoxia can be explained by the activation of the Hif pathway, indicating that it is involved in the regulation of iPC cell generation.

Multi-differentiation potential of iPC cells. To assess the multi-differentiation potential of iPC cells, induced cells were subjected to in vitro induction of differentiation. Established iPC cells in ES culture medium were transferred to DMEM with 10\% FBS (DMEM does not support growth of cells in an undifferentiated state), grown for 20 days to elicit differentiation (post-iPC cells) and their gene expression was then studied by qRT-PCR. The expression of PDXI (a transcription factor involved in pancreatic development), VIMENTIN (a mesenchymal marker) and PAX6 (an ectoderm marker) markedly increased in post-iPC cells derived from wt HCT116 (Fig. 5) and HCT116 p53 null iPC cells (data not shown). The MAP2 expression in post-iPC cells decreased, but remained detectable in wt HCT116 (Fig. 5) and HCT116 p53 null iPC cells (data not shown), although the expression was relatively high in wt HCT116 cells on day 30. Induction of a-Sma (muscle), Gfap (ectoderm), Vimentin, Keratin 19 (epithelial) and Tubb3 (ectoderm) proteins was confirmed by immunocytochemical staining with specific antibodies (data not shown). The data indicate that the four defined factor-induced reprogramming resulted in multi-differentiation potential in cancer cells. Next, a proliferation assay showed that cell growth was significantly 

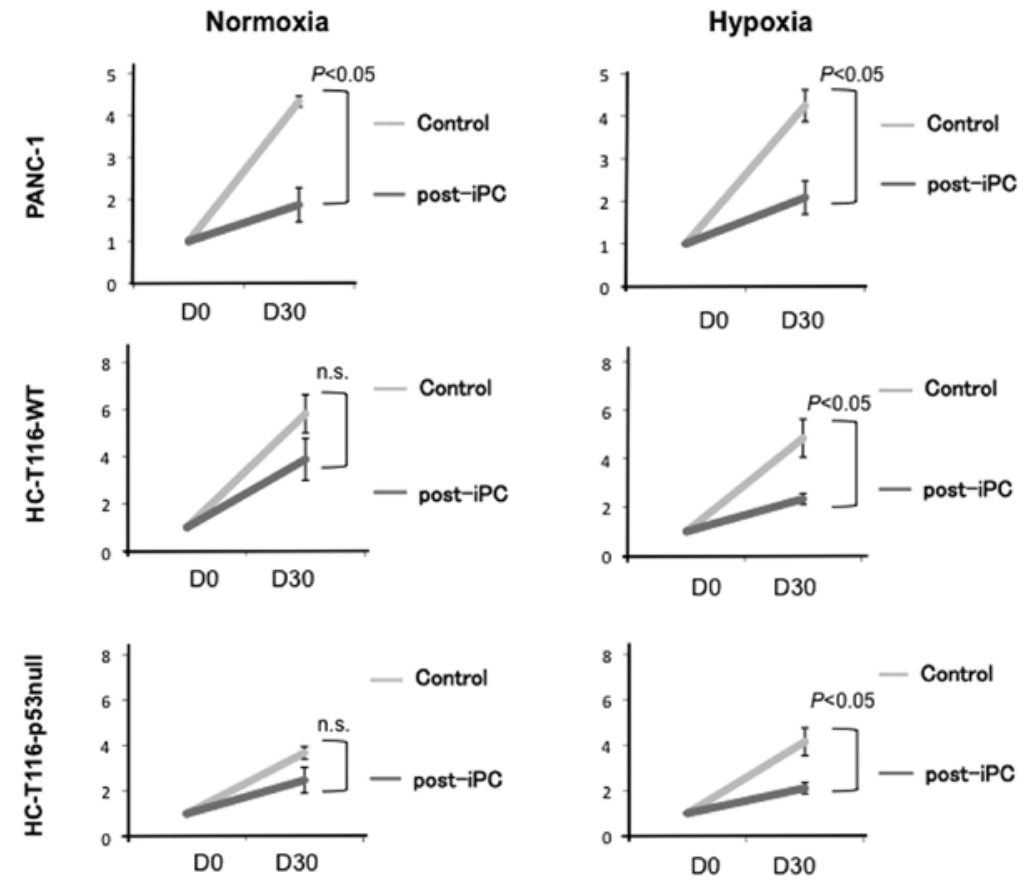

Figure 6. iPC cell proliferation assay. Control parental and iPC cells were derived from wt HCT116, HCT116 p53 null and PANC-1 cells in normoxia or hypoxia and subjected to a proliferation assay. Differences with $\mathrm{p}<0.05$ were considered statistically significant.
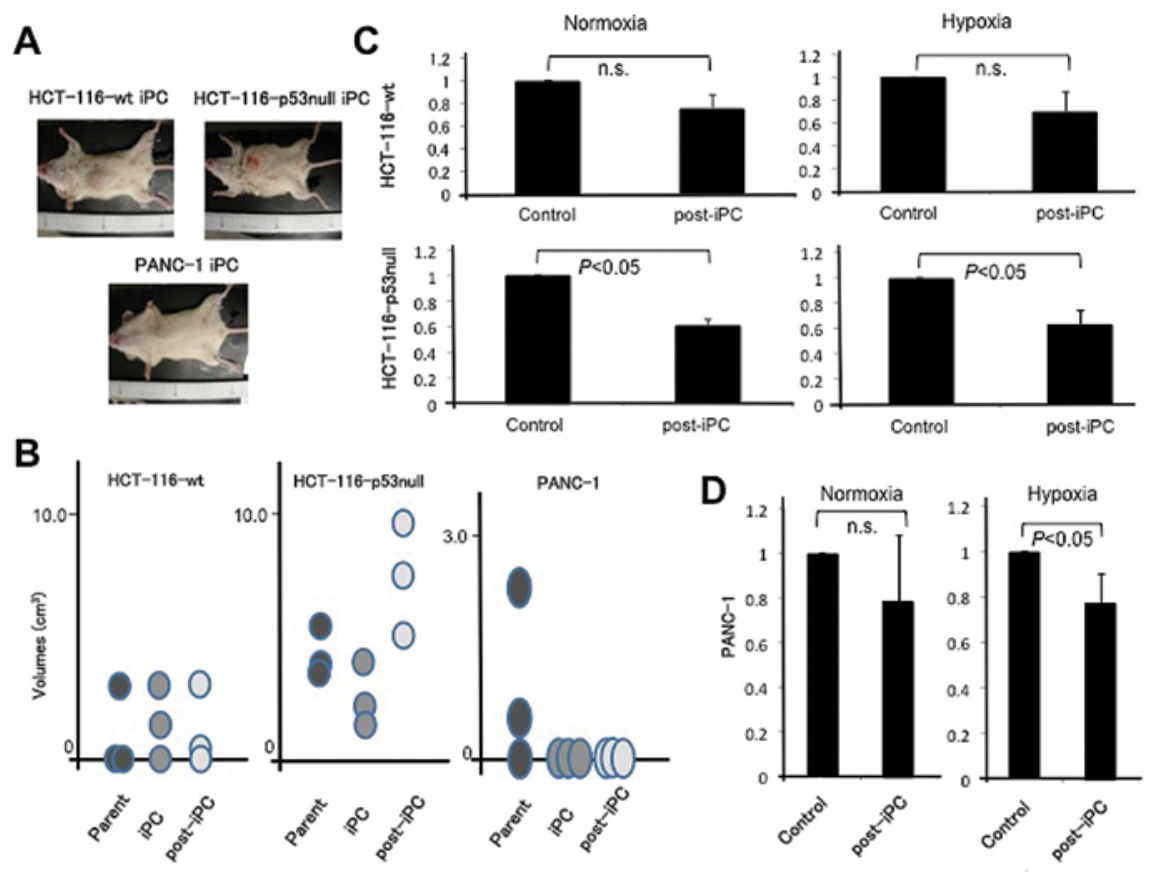

Figure 7. Tumourigenicity and invasion assay of iPC cells. (A and B) Tumourigenicity of parental wt HCT116, HCT116 p53 null and PANC-1 cells and the iPC and post-iPC cells derived from them. (C) Invasion assay of iPC cells derived from wt HCT116 and HCT116 p53 null cells in normoxia and hypoxia. (D) Invasion assay of PANC-1 iPC cells induced in normoxia and hypoxia. Differences with $\mathrm{p}<0.05$ were considered statistically significant.

reduced in post-iPC cells derived from PANC-1 iPC cells, but not parental PANC-1 cells cultured in the same DMEM with $10 \%$ FBS, in both normoxia and hypoxia (p<0.05, Fig. 6). Cell growth was also significantly reduced in wt HCT116 and HCT116 p53 null cells induced in hypoxia $(\mathrm{p}<0.05)$ and, to a lesser extent, in normoxia (not significant; Fig. 6). The data suggested that reprogramming induced a distinct phenotype from parental cancer cells efficiently in hypoxia.
Tumourigenicity and invasion of iPC cells. iPC cells were inoculated into NOD/SCID mice and tumour formation was observed. The tumourigenicity of iPC and post-iPC cells derived from PANC-1 cells was reduced compared to that of parental PANC-1 cells (Fig. 7A and B). The tumourigenicity of iPC and post-iPC cells derived from wt HCT116 cells was comparable to that of parental cells. The tumourigenicity of iPC cells derived from HCT116 p53 null cells was also comparable with that of 
parental HCT116 p53 null cells; however, post-iPC cells derived from HCT116 p53 null cells showed increased tumourigenicity. In vitro invasion assay indicated that tumourigenicity was reduced in iPC cells derived from hypoxia condition and, to a lesser extent, normoxia condition in both wt HCT116 and HCT116 p53 null cells (Fig. 7C), whereas in iPC cells derived from PANC-1 cells the reduction was greater in hypoxia than in normoxia (Fig. 7D). Thus, the present study indicates that induction of iPC cells in hypoxia could cause an enhanced reduction in vitro, while in vivo growth in TP53-deficient background might elicit aggressive transformation and a higher level of tumourigenicity.

\section{Discussion}

Tumours can originate from stem or progenitor cells and epigenetic alterations are involved in cellular differentiation as in $B C R / A B L$ translocation-positive haematopoietic stem cells in chronic myelogenous leukaemia and Lgr5-positive stem cells in CRC (1-3). This suggests that possible corrections of the differentiation program, reversion of aggressive phenotypes or induction of apoptosis in cancer cells may be an ideal therapeutic approach. Reprogramming induction of differentiated cells by transfection of defined transcription factors considerably modifies the epigenetic machinery, but genetic alterations in cancer cannot, in principle, be corrected by the introduction of defined transcription factors. Cancer cells generally harbour genetic instability and the extent to which epigenetic modifications associated with reprogramming can modulate the biological behaviour of tumour cells remains debatable. The present study suggests that the remaining p53 allele in wt HCT116 cancer cells is involved in reprogramming regulation and that its deficiency may accelerate the reprogramming of cancer cells. Since the p53 pathway is altered in $~ 50 \%$ of cancer cells, such cells may be susceptible to reprogramming induction. Furthermore, the present data indicate that hypoxia enhances the reprogramming induction. Considering that cancer metabolism is adaptive to hypoxia glycolysis and that a significant fraction of cancer cells, including cancer stem cells, can presumably survive in hypoxia or in a hypoxic niche, at least two pathways, the Hif and p53 pathways, may be targeted to regulate reprogramming therapy for cancer.

The tumourigenicity assay of HCT $116 \mathrm{p} 53$ null cells indicated that post-iPC cells exhibited a high level of tumour formation in vivo, whereas an in vitro study demonstrated that post-iPC cells derived from HCT116 p53 null cells exhibited reduced invasive activity and a lower cell proliferation rate than control cells. Hypoxia elicited the reprogramming suggesting that the microenvironment of tumour tissues, such as vascularisation in vivo, might affect tumour activation and that tumour activation is not a cell-autonomous mechanism. The p53 protein has been shown to limit angiogenesis, at least partly, by interfering with the central regulators of hypoxia that mediate angiogenesis (reviewed in ref. 18). Tumours with inactivated tumour suppressor p53 function, approximately half of all tumours, appear more vascularised, are often more aggressive and are correlated with poor post-treatment prognosis. Thus, the loss of functional p53 during tumourigenesis represents an essential step in the switch in hypoxia to an angiogenic phenotype that characterises aggressive tumours (18). To inhibit malignant transformation observed in the reprogrammed TP53-deficient cancer cells (the present study) and in other cancer cells with gain-of-function mutations such as TP53 $3^{\mathrm{R} 175 \mathrm{H}}$ and $\mathrm{KRAS}^{\mathrm{G} 12 \mathrm{D}}$ (19), we suggest that the combination of anti-angiogenic therapy and therapies targeting TP53 deficiency may be beneficial. For example, anti-vascular endothelial growth factor receptor antibodies may suppress the deleterious effect of TP53-deficient reprogramming. We speculate that, although TP53 overlapped with the hypoxia pathway, TP53 may play a critical role in the surveillance of the malignant phenotype or deleterious mutations in reprogrammed cancer cells.

Our results suggest a specific target for reprogramming in the hypoxia pathway. The expression of Hif $2 \alpha$ is celltype specific and its biological role is distinct from that of Hif $1 \alpha$. While Hif $1 \alpha$ is ubiquitously expressed, Hif $2 \alpha$ is more prominently detected in vascular endothelial cells during embryonic development and is indeed an upstream regulator of OCT3/4 (15). Furthermore, in addition to being present in endothelial cells, HIF $2 \alpha$ mRNA has been detected in kidney fibroblasts, liver hepatocytes, epithelial cells of the intestinal lumen, pancreatic interstitial cells and other interstitial cells $(20,21)$. In this study, the effect of introducing ES-like genes in gastrointestinal cancer cells may have been enhanced by hydroxylation-defective mutation of $H I F 2 \alpha$ and, to a lesser extent, HIFla, suggesting that cell type-specific modifications involving the use of different Hif subunits could be a reliable method of fine-tuning reprogramming efficiency. To the best of our knowledge, this is the first report demonstrating involvement of the Hif family in reprogramming and iPC cell generation.

\section{Acknowledgements}

We thank Dr Bert Vogelstein (Johns Hopkins University, Baltimore, MD) for providing TP53-deficient cell lines. This work was partly supported by a grant from the Core Research for Evolutional Science and Technology (CREST) (H.I., M.M.), a Grant-in-Aid for Scientific Research on Priority Areas (M.M.), a Grant-in-Aid for Scientific Research from the Ministry of Education, Culture, Sports, Science and Technology (H.I., M.M.), a Grant-in-Aid for the 3rd Comprehensive 10-year Strategy for Cancer Control Ministry of Health, Labour and Welfare (H.I., M.M.), a grant from the Tokyo Biochemical Research Foundation (M.M.) and a grant from the Princess Takamatsu Cancer Research Fund, Japan (H.I.).

\section{References}

1. Tenen DG: Disruption of differentiation in human cancer: AML shows the way. Nat Rev Cancer 3: 89-101, 2003.

2. Chen J, Odenike O and Rowley JD: Leukaemogenesis: more than mutant genes. Nat Rev Cancer 10: 23-36, 2010.

3. Markowitz SD and Bertagnolli MM: Molecular origins of cancer: Molecular basis of colorectal cancer. N Engl J Med 361: 2449-2460, 2009.

4. Jones PA and Baylin SB: The fundamental role of epigenetic events in cancer. Nat Rev Genet 3: 415-428, 2002.

5. Piekarz RL and Bates SE: Epigenetic modifiers: basic understanding and clinical development. Clin Cancer Res 15: 3918-3926, 2009.

6. Takahashi K and Yamanaka S: Induction of pluripotent stem cells from mouse embryonic and adult fibroblast cultures by defined factors. Cell 126: 663-676, 2006.

7. Yamanaka S: Elite and stochastic models for induced pluripotent stem cell generation. Nature 460: 49-52, 2009. 
8. Miyoshi N, Ishii H, Nagai K, et al: Defined factors induce reprogramming of gastrointestinal cancer cells. Proc Natl Acad Sci USA 107: 40-45, 2010.

9. Gil J and Peters G: Regulation of the INK4b-ARF-INK4a tumour suppressor locus: all for one or one for all. Nat Rev Mol Cell Biol 7: 667-677 2006

10. Zhao Y, Yin X, Qin H, et al: Two supporting factors greatly improve the efficiency of human iPSC generation. Cell Stem Cell 3: 475-479, 2008.

11. Kawamura T, Suzuki J, Wang YV, et al: Linking the p53 tumour suppressor pathway to somatic cell reprogramming. Nature 460 : 1140-1144, 2009.

12. Hong H, Takahashi K, Ichisaka T, et al: Suppression of induced pluripotent stem cell generation by the p53-p21 pathway. Nature 460: 1132-1135, 2009.

13. Yoshida Y, Takahashi K, Okita K, Ichisaka T and Yamanaka S: Hypoxia enhances the generation of induced pluripotent stem cells. Cell Stem Cell 5: 237-241, 2009.

14. Yeung SJ, Pan J and Lee MH: Roles of p53, MYC and HIF-1 in regulating glycolysis - the seventh hallmark of cancer. Cell Mol Life Sci 65: 3981-3999, 2008

15. Poon E, Harris AL and Ashcroft M: Targeting the hypoxiainducible factor (HIF) pathway in cancer. Expert Rev Mol Med 11: e26, 2009.
16. Ben-Porath I, Thomson MW, Carey VJ, Ge R, Bell GW, Regev A and Weinberg RA: An embryonic stem cell-like gene expression signature in poorly differentiated aggressive human tumors. Nat Genet 40: 499-507, 2008

17. Takahashi K, Tanabe K, Ohnuki M, Narita M, Ichisaka T, Tomoda $\mathrm{K}$ and Yamanaka S: Induction of pluripotent stem cells from adult human fibroblasts by defined factors. Cell 131: 861-872, 2007

18. Teodoro JG, Evans SK and Green MR: Inhibition of tumor angiogenesis by p53: a new role for the guardian of the genome. J Mol Med 85: 1175-1186, 2007.

19. Nagai K, Ishii H, Miyoshi N, et al: Long-term culture following ES-like gene-induced reprogramming elicits an aggressive phenotype in mutated cholangiocellular carcinoma cells. Biochem Biophysic Res Commun 395: 258-263, 2010.

20. Rosenberger C, Mandriota S, Jürgensen JS, et al: Expression of hypoxia-inducible factor-1alpha and -2alpha in hypoxic and ischemic rat kidneys. J Am Soc Nephrol 13: 1721-1732, 2002.

21. Wiesener MS, Jürgensen, JS, Rosenberger C, et al: Widespread hypoxia-inducible expression of HIF-2alpha in distinct cell populations of different organs. FASEB J 17: 271-273, 2003. 